\title{
Serum thymidine kinase 1 reflects the progression of pre- malignant and malignant tumors during therapy
}

\author{
XIAO-HONG XU ${ }^{1}$, YI-MIN ZHANG ${ }^{1}$, XIN-HUA SHU ${ }^{1}$, LU-HU SHAN $^{1}$, ZI-WEI WANG $^{2}$, \\ YONG-LIE ZHOU ${ }^{3}$, HUAI-KAI WEN ${ }^{4}$, FAN HE ${ }^{5}$, ELLEN HE ${ }^{6}$ and SVEN SKOG ${ }^{6}$
}

\begin{abstract}
${ }^{1}$ Department of Clinical and Laboratory Medicine, Zhejiang Cancer Hospital; ${ }^{2}$ The Central Laboratory, Hangzhou First People's Hospital; ${ }^{3}$ Department of Clinical and Laboratory Medicine, Zhejiang Provincial People's Hospital; ${ }^{4}$ Department of Clinical and Laboratory Medicine, The Second Affiliated Hospital of Wenzhou Medical College;

${ }^{5}$ Zhejiang Centre for Disease Control, P.R. China; ${ }^{6}$ Department of Oncology, Karolinska Institute, Sweden
\end{abstract}

Received May 26, 2008; Accepted July 7, 2008

DOI: $10.3892 / \mathrm{mmr}_{-} 00000017$

\begin{abstract}
This study evaluated the clinical utility of serum thymidine kinase 1 (STK1) in following the progression of pre-malignancies and malignancies and in monitoring the response of common carcinomas to therapy within a routine clinical setting. The STK1 concentration levels of patients with malignancies $(n=224)$, pre-malignancies $(n=10)$, non-tumor/ non-proliferating diseases (systemic lupus erythematosus, SLE) $(n=53)$, benign tumors $(n=20)$ and healthy volunteers $(n=761)$ were determined by enhanced chemoluminescence dot blot assay. Prior to treatment, STK1 levels in the pre-malignant group alone $(3.1 \pm 2.3)$ or in the pre-malignant and malignant groups together $(2.3 \pm 1.9)$ were significantly higher than in the benign $(1.4 \pm 0.8)$, SLE $(1.1 \pm 0.8)$ or healthy volunteer $(0.6 \pm 0.4)$ groups $(\mathrm{p}<0.01)$. According to ROC analysis, the STK1 assay provided a high degree of discrimination between STK1positive pre-malignant (0.978) or pre-malignant + malignant (0.941) patients and STK1-negative healthy individuals. After varying treatments (surgery, chemotherapy, X-ray), STK1 levels increased by $40-50 \%$ during the first month, then decreased back to normal values or even lower. Following treatment, STK1 levels were significantly increased in squamous cell carcinoma (SCC) as compared to adenocarcinoma (AC) patients. In other types of malignancies, STK1 levels decreased from as early as the first month. The STK1 levels of relapsed treated patients were significantly higher (50-60\%) than those of mid/long-term treated patients. In conclusion, the
\end{abstract}

Correspondence to: Professor Sven Skog, Eksätravägen 140, 75655 Uppsala, Sweden

E-mail:svenisak@hotmail.com

Professor Xiao-Hong Xu, Department of Clinical and Laboratory Medicine, Zhejiang Cancer Hospital, Hangzhou, P.R. China E-mail: zjhzxxh@163.com

Key words: thymidine kinase 1, serum, cancer, squamous cell carcinoma, adenocarcinoma
STK1 assay discriminated between patients with malignancies and healthy individuals very well, and is therefore potentially useful for a broad range of clinical applications. For example, it could be used for the evaluation of early tumor progression or of tumor progression during therapy in routine clinical settings, as well as for the screening of healthy individuals.

\section{Introduction}

Neoplasia is the abnormal, uncoordinated and excessive growth of cells. Since 1960, a number of potential sensitive and specific serum markers have been investigated for their utility in the detection of invisible tumors, i.e., inaccessible malignancies occurring ahead of imaging or palpable assessment. Examples of such serum markers are CEA, CA19.9 (1-3), CA125 (4), AFP (5) and PSA (6), all of which are widely used in clinical practice today. Another example is CA15.3, which has proven useful in patients with metastatic breast cancer, when response to systemic therapy cannot be assessed $(4,7)$. The combination of TPS, CA15-3 and IGFBP-3 increases sensitivity to $85 \%$, and thus improves predictive ability (8). Increased CEA concentrations reflect higher risk in patients with adenocarcinomas (ACs), but not in those with squamous cell carcinomas (SCCs). CA125 levels are associated with a worse prognosis due to an increased possibility of relapse (4). Serum CA125 is an imperative indicator of ovarian carcinoma malignancies. In 2006, ASCO recommended that CEA should be used pre-operatively in colorectal cancer (9) to assist in staging and surgical planning. CEA is the marker of choice for monitoring the response of metastatic disease to systemic therapy. If the patient is a potential candidate for surgery or chemotherapy, in those with stage II and III tumors the determination of post-operative CEA levels should be performed every 3 months for at least 3 years. For pancreatic cancer, it is recommended that CA 19-9 be determined every 1-3 months in patients with locally advanced or metastatic diseases receiving active therapy. However, the predictive ability of serum tumor markers for response to anticancer therapy is still controversial, and an accurate appraisal of their individual characteristics must be performed (6). Furthermore, multivariate analysis of different studies show that serum tumor markers fluctuate more than imaging techniques. 
Thymidine kinase 1 (TK1) is a pyrimidine metabolic pathway enzyme involved in salvage DNA synthesis, and is thus a cell cycle-dependent marker. Serum thymidine kinase 1 (STK1) levels in malignant cells are proportional to cellular proliferation rates (10-13). Thymidine kinase activity has been used as a proliferation marker since 1980 in serum and cytosol fractions of tissues. It is almost undetectable in normal serum (14-16), but increases to varying degrees in malignant tumors, depending on their type, stage (16), whether they are fast or slow growing (17), and whether they are treated or untreated (18). In a clinical study of 1,692 breast cancer patients (19), TK1 activity in the cytosol was correlated to a shorter survival time and poor outcome using endocrine treatment (tamoxifen) (20). Furthermore, commercially available anti-TK1 antibodies have recently provided an attractive alternative in clinical cancer applications. In patients with solid tumors, STK1 concentrations are a more sensitive and reliable marker than TK activity $(18,21)$. He et al reported that STK1 levels were associated with relapse, while TK activity and CA15-3 were not (18).

In this study, we examined the utility of STK1 in the prognosis of pre-malignant and malignant tumors of common carcinomas and in monitoring the results of therapy within routine clinical settings. The ability of the STK1 assay to discriminate between malignant and non-malignant groups was tested by ROC analysis. Healthy individuals were also tested. As a reference, we additionally examined the serum levels of individuals with benign tumors and serum from those with a non-proliferating disease.

\section{Materials and methods}

Patients. Serum samples from patients with malignant tumors of common carcinomas $(n=234)$ and with benign tumors $(n=20)$ were colleted from unselected cases in routine clinical settings, including pre-operation, post-operation and/or chemotherapy in combination with operation, at the Hanzhou Cancer Hospital, P.R. China, in 2007. All patients had histologically-diagnosed tumors, which were prospectively evaluated as pre-malignant (cervical, 10) and malignant (NPC, 33; cervical, 32; lung, 25; ovarian, 24; esophageal, 22; gastric, 16; breast, 14; colon, 10; rectum, 9; liver, 7; lymphoma, 7; other, 25). A few of the patients with malignant tumors had their clinical stage, determined according to the AJCC Cancer Staging Manual (22), recorded as follows: stage II, $\mathrm{n}=11$; III, $\mathrm{n}=14 ; \mathrm{IV}, \mathrm{n}=22$. The majority of the patients had advanced malignant tumors. Adjuvant chemotherapy was the standard treatment for clinical management, administered as follows for the varying types of cancer: lung, GP strategy 3 weeks (gemcitabine $1250 \mathrm{mg} / \mathrm{m}^{2}$, cisplatin $75 \mathrm{mg} / \mathrm{m}^{2}$ ) or NP strategy 3 weeks (vinorelbine $25 \mathrm{mg} / \mathrm{m}^{2}$, cisplatin $75 \mathrm{mg} / \mathrm{m}^{2}$ ); intestinal, FOLFOX6 strategy 2 weeks (oxaliplatin $100 \mathrm{mg} / \mathrm{m}^{2}$, leucovorin $400 \mathrm{mg} / \mathrm{m}^{2}$, fluorouracil $400 \mathrm{mg} / \mathrm{m}^{2}$, fluorouracil $2400 \mathrm{mg} / \mathrm{m}^{2}$ ); breast, FEC strategy 4 weeks (cyclophosphamide $500 \mathrm{mg} / \mathrm{m}^{2}$, epirubicin $50 \mathrm{mg} / \mathrm{m}^{2}$, fluorouracil $500 \mathrm{mg} / \mathrm{m}^{2}$ ); gastric, FOLFOX4 strategy 2 weeks (oxaliplatin $85 \mathrm{mg} / \mathrm{m}^{2}$, leucovorin $200 \mathrm{mg} / \mathrm{m}^{2}$, fluorouracil $400 \mathrm{mg} / \mathrm{m}^{2}$, fluorouracil $600 \mathrm{mg} / \mathrm{m}^{2}$ ); esophageal, PF strategy 4 weeks (cisplatin $75 \mathrm{mg} / \mathrm{m}^{2}$, fluorouracil $1000 \mathrm{mg} / \mathrm{m}^{2}$ ); pancreatic, GEM+xeloda strategy 3 weeks (gemcitabine $1000 \mathrm{mg} / \mathrm{m}^{2}$, capecitabine $1000 \mathrm{mg} / \mathrm{m}^{2}$ ); ovarian, TP strategy 3 weeks (taxol $175 \mathrm{mg} / \mathrm{m}^{2}$, cisplatin $75 \mathrm{mg} / \mathrm{m}^{2}$ ); cervical, TP strategy 3 weeks (taxol $135 \mathrm{mg} / \mathrm{m}^{2}$, cisplatin $75 \mathrm{mg} / \mathrm{m}^{2}$ ). For radiotherapy, standard treatments for the different types of malignancy were administered.

For comparative purposes, patients with benign tumors, non-tumor non-proliferating disease, systemic lupus erythematosus (SLE) with a positive reaction and healthy individuals were investigated in parallel. SLE is an immunological abnormality with a multisystem disorder. Serum samples were colleted from 43 patients positive for SLE at the Zhejiang People's Hospital. Sera samples from 761 healthy individuals with no evidence of contagious or cancerous disease were colleted at the Zhenjiang People's Hospital, Wenzhou Affinity Second Hospital, Wenzhou Medical Institute and Hanzhou Zhiyuan Health Centre. The healthy individuals worked in restaurants or hotels as waitresses and waiters. The present study of serum TK1 was conducted in accordance with the Helsinki Declaration of 1983.

ECL dot blot assay. ECL dot blot assay was performed according to the manufacturer's protocol (commercial kit, SSTK Inc., Shenzhen, P.R. China). Blood samples (2 ml) were collected between 7 and 9 a.m. from individuals who had not eaten any breakfast. The drawn venous blood was stored for 2-3 h at room temperature (RT) in non-heparin tubes, then centrifuged at 4,000 rpm for 8-10 min and stored at $-20^{\circ} \mathrm{C}$ until analysis. Three $\mu 1$ of serum were directly applied onto a nitrocellulose membrane (HybandTM-C, Amersham). TK1 standards (20, 6.6, 2.2 pM) were used as an extrapolation standard. The membrane was blocked in TBS (Tris-buffered saline) with $6 \%$ non-fat milk for $1 \mathrm{~h}$ and incubated at RT for $2 \mathrm{~h}$ after the addition of primary anti-TK1 antibody. After incubation with a biotinylated secondary antibody for $40 \mathrm{~min}$ at RT, the membrane was incubated in TBS buffer with avidin-HRP-streptavidin, followed by the addition of ECL substrate. The light intensity of a single spot on the membrane was detected using a CIS-1 imaging system (SSTK Inc.). Based on the light intensities of the known concentrations of TK1 standards, the light intensities of the serum TK1 spots were re-calculated and expressed as pM. The sensitivity of the assay was $0.3 \mathrm{pM}$, its reliability $98 \%$ and the CV-value of the duplicates $9.4 \%$. All experiments were performed in a blinded manner and in duplicate. The TK1 antibody has been biochemically characterized (23). Western blot analysis of TK1-positive cells (CEM wild-type) and serum from patients with gastric cancer showed one single band corresponding to the native form of TK1. In TK1negative cells (CEM-) and in serum from healthy individuals with no detectable levels of TK1 (dot blot assay), no band was apparent.

Statistical calculations. The mean value of STK1 levels was calculated by a means \pm standard deviation program. For the comparison of STK1 concentration levels among several groups, the Kruskal-Wallis test was used, while the Nemenyi test and Bonferroni correction were used when two groups were compared. Data analysis was performed using SPSS software 15.0. Differences were considered significant when the $\mathrm{p}$-value was $<0.05$. 
Table I. STK1-positive cases and concentration levels in patients with pre-malignant and malignant tumors prior to treatment, in patients with benign tumors, in systemic lupus erythematosus patients, and in healthy volunteers.

\begin{tabular}{|c|c|c|c|c|c|}
\hline Group & Total no. cases & No. TK1 positive (\%) & STK1 (pM) & $\chi^{2}$ value & P-value \\
\hline Pre-malignant & 10 & $7(70.0)$ & $3.1 \pm 2.3$ & & \\
\hline Malignant & 224 & $88(39.3)$ & $2.3 \pm 1.9$ & & \\
\hline Benign tumor & 20 & $3(15.0)$ & $1.4 \pm 0.8$ & 456.56 & $<0.0001$ \\
\hline Systemic lupus erythematosus & 53 & $4(7.6)$ & $1.1 \pm 0.8$ & & \\
\hline Healthy volunteers & 761 & $7(0.9)$ & $0.6 \pm 0.4$ & & \\
\hline
\end{tabular}

Statistical analysis performed using the Kruskal-Wallis test. Concentration levels are the mean values \pm standard deviation.

Table II. Comparison of STK1 concentration levels in patients with pre-malignant and malignant tumors prior to treatment, in patients with benign tumors, in systemic lupus erythematosus patients, and in healthy volunteers.

\begin{tabular}{lccr}
\hline Group & No. cases & STK1 (pM) & $\chi^{2}$ value \\
\hline Pre-malignant + malignant (pre-treatment) & 91 & $2.5 \pm 2.0$ & \\
Benign tumor & 20 & $1.4 \pm 0.8$ & 285.17 \\
Systemic lupus erythematosus & 53 & $1.1 \pm 0.8$ & $<0.0001$ \\
Healthy volunteers & 761 & $0.6 \pm 0.4$ & \\
\hline
\end{tabular}

Statistical analysis performed using the Nemenyi test. Concentration levels are the mean values \pm standard deviation.

\section{Results}

The STK1 levels of patients with pre-malignant, malignant, and benign tumors, patients with non-tumor/non-proliferating diseases (SLE-positive) and healthy volunteers are shown in Table I. The number of STK1-positive cases was determined according to a cut-off value of $2 \mathrm{pM}$.

STK1-positive patients. The number of STK1-positive patients was, at $\sim 70 \%$, highest in the pre-malignant group. The number of positive malignant patients was $\sim 40 \%$ (Table I). In the benign and SLE groups, STK1-positive patients comprised just 15 and $8 \%$, respectively, while the value for healthy individuals was $<1 \%$ (Table I).

Concentration of TK1 in serum. The concentrations of STK1 in the pre-malignant $(3.1 \pm 2.3 \mathrm{pM})$ and malignant $(2.3 \pm 1.9 \mathrm{pM})$ groups were higher than in the benign tumor $(1.4 \pm 0.8 \mathrm{pM})$, SLE $(1.1 \pm 0.8 \mathrm{pM})$ and healthy volunteer $(0.6 \pm 0.4 \mathrm{pM})$ groups, with statistical significance $(\mathrm{p}<0.0001)$ (Table I). The mean concentration of STK1 from the pre-malignant + malignant groups $(2.5 \pm 2.0 \mathrm{pM})$ was also higher than in the other groups, with statistical significance $(\mathrm{p}<0.001)$ (Table II). One patient with lung carcinoma and one with NPC 3 months after chemo/ radiotherapy showed marked remission of the tumor disease, with STK1 levels of 0.6 and $0.7 \mathrm{pM}$, respectively.

ROC analysis. The specificity and sensitivity of the STK1 dot blot assay were tested by receiver operating characteristic
(ROC) curve analysis. A graphical approach, where the sensitivity as $\mathrm{Y}$ is plotted versus the fraction of false positives (the false positive rate) as $\mathrm{X}$ for each possible cut-off value, was employed in order to create the ROC curve. In this study, sensitivity was plotted versus 1 -specificity (false positive) (Figs. 1 and 2) reflecting the binary classifier system, as its discrimination threshold is varied. The area under the ROC curve shows the reliability of the STK1 assay when used to discriminate between healthy individuals and patients with malignancies. A value $>0.9$ means that the STK1 assay is a highly reliablity test.

In the present study, we tested the reliability of the STK1 assay in a group with pre-malignancies (Fig. 1) and in a group with pre-malignancies + malignancies together (Fig. 2). The area under the ROC curve for pre-malignant patients was 0.978 and for the pre-malignant + malignant patients 0.941 .

STK1 concentration levels during therapy. Changes in STK1 concentration levels during the treatment of patients with the SCC and AC carcinoma types and of patients with other types of carcinoma were divided into the following two groups according to the duration of treatment (Table III): short-term, $<1$ month after operation or single chemotherapy, with chemotherapy accounting for $1 / 3$ of the total doses administered; mid/long-term, $>1$ month after operation or multichemotherapy, with chemotherapy accounting for $>2 / 3$ of the total doses administered (Tables III and IV). A third group of patients was also included, indicating the STK1 concentration levels of patients who had undegone a relapse (Table III). 


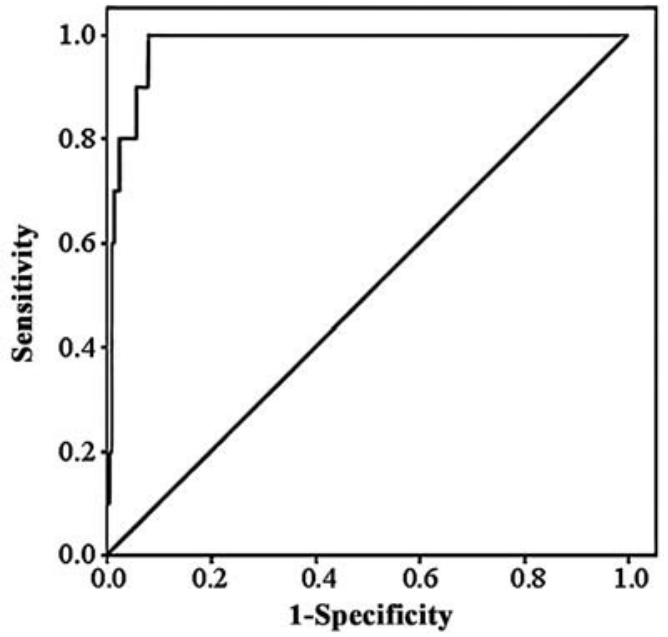

Figure 1. ROC curve. A plot of the STK1 levels of the healthy group in comparison to those of the untreated pre-malignant group.

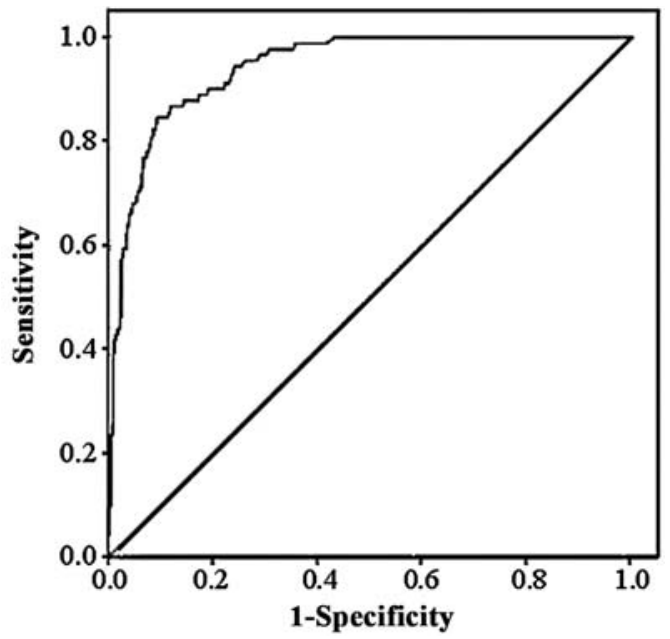

Figure 2. ROC curve. A plot of the STK1 levels of the healthy group in comparison to those of the untreated pre-malignant + malignant groups.

Table III. STK1 concentration levels of squamous cell carcinoma, adenocarcinoma and other types of carcinoma during therapy.

\begin{tabular}{|c|c|c|c|c|c|c|c|c|}
\hline & \multicolumn{2}{|c|}{ Prior to treatment } & \multicolumn{2}{|c|}{ Short-term treatment } & \multicolumn{2}{|c|}{ Mid/long-term treatment } & \multicolumn{2}{|c|}{ Relapse } \\
\hline & Levels & $\%$ (no.) patients & Levels & $\%$ (no.) patients & Levels & $\%$ (no.) patients & Levels & $\%$ (no.) patients \\
\hline SCC & $2.4 \pm 2.3$ & $34.2(41)$ & $3.5 \pm 2.1$ & $72.7(11)$ & $2.4 \pm 2.4$ & $38.7(31)$ & $3.7 \pm 1.9$ & $77.8 \quad(9)$ \\
\hline $\mathrm{AC}$ & $2.0 \pm 1.3$ & $38.7(31)$ & $2.8 \pm 1.3$ & $61.1(18)$ & $1.4 \pm 1.0$ & $10.0(40)$ & $2.3 \pm 1.9$ & $40.0(15)$ \\
\hline Other & $3.6 \pm 1.9$ & $77.8 \quad(9)$ & $3.2 \pm 1.8$ & $66.7 \quad(3)$ & $1.8 \pm 2.0$ & $23.0(13)$ & $2.5 \pm 0.8$ & $66.7 \quad(3)$ \\
\hline
\end{tabular}

Short-term treatment, within a month of the start of the treatment; mid/long-term treatment, after one month of treatment. Concentration levels are the mean values \pm standard deviations. SCC, squamous cell carcinoma; AC, adenocarcinoma.

Table IV. Variance analysis of STK1 concentration levels in patients with squamous cell carcinoma, adenocarcinoma and other types of carcinoma during therapy.

\begin{tabular}{|c|c|c|c|c|c|}
\hline Variation & $\begin{array}{l}\text { Sum of squares } \\
\text { of mean deviation }\end{array}$ & $\begin{array}{c}\text { Degree } \\
\text { of freedom }\end{array}$ & $\chi^{2}$ value & $\mathrm{F}$ & P-value \\
\hline$<1$ month vs. $>1$ month treatment time & 49.485 & 3 & 16.495 & 4.973 & 0.002 \\
\hline SCC vs. AC & 32.039 & 2 & 16.019 & 4.829 & 0.009 \\
\hline Error & 723.127 & 218 & 3.317 & & \\
\hline Total & 800.57 & 224 & & & \\
\hline
\end{tabular}

Statistical analysis performed using Bonferroni correction. SCC, squamous cell carcinoma; AC, adenocarcinoma.

Table V. Age distribution of patients with pre-malignant and malignant cervical tumors in relation to STK1 concentration levels.

\begin{tabular}{llcccc}
\hline Tumor progression & \multicolumn{2}{c}{ Type (No.) } & Age range (years) & Mean age (years) & STK1 (pM) \\
\hline Pre-malignant & Cervical & $(10)$ & $31-44$ & 37 & $3.1 \pm 2.3$ \\
Malignant & Cervical & $(30)$ & $33-71$ & 48 & $2.4 \pm 2.0$ \\
Malignant & Mixed tumor (224) & $33-74$ & 53 & $2.3 \pm 1.9$ \\
\hline
\end{tabular}

Concentration levels are the mean values \pm standard deviations. 
The results indicate that the STK1 concentration levels of both the SCC and AC groups, and of the groups with other kinds of tumors, changed during treatment, with statistical significance $(\mathrm{p}<0.002)$. Using Bonferroni correction, the untreated and short-term-treated groups were statistically different $(\mathrm{p}<0.002)$, as were the short-term-treated and mid/long-termtreated groups $(p<0.002)$. STK1 concentration levels in the relapsed group were significantly higher than in the mid/ long-term-treated group $(\mathrm{p}<0.002)$. Additionally, the STK1 concentration levels of patients with SCC were significantly higher than those of patients with $\mathrm{AC}(\mathrm{p}<0.01)$.

STK1 concentration levels in relation to age. The frequency of malignancies starts to increase at around the age of 50 years, and accelerates year by year. Here, we were able to compare the age distribution of the patients with pre-malignant and malignant cervical tumors, and to relate the results to STK1 concentration (Table V). As mentioned above (Table I), 70\% of patients with pre-malignancy were STK1-positive. These pre-malignant cervical patients were also younger (mean age 37) than patients with malignant cervical cancer (mean age $48)$, with statistical significance $(\mathrm{p}<0.001)$. The mean age of the group of patients with different types of malignancy was 53 years (Table V).

\section{Discussion}

Thymidine kinase (TK, ATP: thymidine 5-phosphotransferase, EC.2.7.1.21), an enzyme of the pyrimidine salvage pathway, catalyzes the phosphorylation of thymidine to thymidine monophosphate (24). The TK enzyme in human cells appears in two isoenzyme forms, a cytosolic (TK1) and a mitochondrial (TK2) form. TK1 is part of the regulation of the intracellular thymidine pool. The level of TK1 rises at the G1/S-phase boundary and increases up to the late S/early G2 phases of the cell cycle. TK1 is present in proliferating normal and tumor cells, but is virtually absent from quiescent cells $(12,13)$. It is of considerable interest in clinical settings because its level is highly dependent on cell growth. TK1 is a sensitive and specific marker for prognosis $(16,18,19)$, for the monitoring of the outcome of cancer therapy (25-28), and for the screening of healthy individuals for the risk of malignant disease (unpublished data).

The usefulness of a tumor marker depends on its ability to distinguish between normal healthy individuals and patients with malignancies. One way to determine its ability to discriminate between the two is by using ROC analysis; the area under the ROC curve indicates whether the tumor marker is valuable or not. ROC analysis of the STK1 assay in the present study resulted in an area under the curve above 0.9 , which indicates that the STK1 assay is a test with high discrimination between healthy individuals and patients with pre-malignancies or malignancies. This is in accordance with previous results (21).

Of the various degrees of malignancy, pre-malignancy is of particular interest, since discovery at this stage considerably increases the chances of curing the patient. In the present study, we found approximately $70 \%$ of pre-malignant cervical cancer to be STK1-positive. We also found that the mean age of pre-malignant cervical cancer patients was approximately 10 years below that of patients with cervical malignancy.
This time difference between the appearance of pre-malignancy and the appearance of malignancy is in accordance with results from breast cancer. The Cancer Committee of the College of American Pathologists has stated that the presence of atypical ductal hyperplasia of the breast increases the risk of the subsequent (in 8.3 years) development of invasive breast cancer by 4.0-5.0 times (29). WHO has also reported that the time to developing a breast carcinoma in situ from a benign tumor, over an atypical breast, is approximately 10 years (29). Consequently, although the number of cervical cancer patients was relatively low in this study, in our opinion it is likely that STK1 is a useful tool for the discovery of premalignancies. However, further studies are needed to confirm this observation.

In accordance with recent studies on STK1, we found that STK1 levels were significantly higher in patients with relapse than in non-relapsed patients after operation/adjuvant therapy. Thus, STK1 concentrations again proved to be a reliable index for monitoring the results of cancer treatment in patients, capable of distinguishing between patients with or without remaining malignant tumors. As a result, increased STK1 levels could be used not only for the evaluation of tumor progression, but also in order to evaluate the effectiveness of therapy. Elevated levels of STK1 in patients with recurrence after curative surgery/adjuvant therapy provide new avenues for evaluating therapy in the short term. It also makes it possibile to pay special attention to patients at risk of recurrence during early post-operative surveillance. STK1 also allows for an improvement in treatment, and can probably precede imaging techniques resulting in early pre-treatment, enabling increased survival chances and a better quality of life for cancer patients.

It is of interest to determine whether STK1 levels differ between patients with squamous cell carcinoma and adenocarcinoma, since these patients may be receiving different types of therapy. Therefore, the significant difference found between the STK1 levels of these two groups is of clinical import, as it may provide a new tool for the selection of therapeutic strategy.

Although the mean STK1 level of patients with benign tumors was below the cut-off value of $2.0 \mathrm{pM}, 15 \%$ of these patients were STK1-positive. This result is in accordance with recent studies (30). STK1-positive benign patients may be on the way to developing pre-malignancies/malignancies, as the Cancer Committee of The College of American Pathologists and WHO have reported an elevated risk of benign tumors developing into malignancies (29).

TK1 is a proliferation marker and, in addition to being found in malignant diseases, is found in elevated levels at an acute stage of certain viral infections (31), such as hepatitis A (32), hepatocirrhosis (21) and HIV-infected patients (33), as well as among patients with anaemia (34) or inflammation (21). Therefore, when evaluating STK1 results, it is necessary to examine possible causes for elevated STK1 other than malignancies (30).

To this end, we investigated a non-tumor/non-proliferating disease, systemic lupus erythematosus (SLE), to see whether this type of disease affects STK1 levels. The STK1-positive rate of SLE patients was only $7.6 \%$, significantly lower than that of the pre-malignant/malignant groups. Thus, it can be 
concluded that this disease does not incite elevated levels of STK1. The main clinical features of SLE are skin rashes, light sensitivity, arthritis, anaemia, leucopenia and renal disease. One of the STK1-positive (3.4 pM) SLE patients may have had anaemia or another proliferating disease.

Currently, no reliable screening methods for cancer in healthy individuals exist, with the exception of PSA for prostate and mammography for breast cancer. In the long-term prediction of prostate cancer, PSA gives elevated false positive values, but can predict subsequent (25 years later) advanced cancer if tested prior to the age of 50 years (6). The mean value of STK1 was $0.6 \pm 0.4 \mathrm{pM}$, a similar range to that reported by He et al (21). Of the 761 healthy individuals tested in the present study, $0.9 \%$ (7/761) were STK1-positive. Two of these seven had a STK1 level just above the cut-off value of $2.0 \mathrm{pM}$. Considering that the STK1 assay has a CV of approximately $10 \%$, we have 5 accurate STK1-positive cases, corresponding to approximately $0.7 \%(5 / 761)$ of STK1-positive healthy individuals. This value is somewhat higher than that expected based on the cancer incidence rate of $0.2 \%$ in P.R. China (35) and of $0.4-0.5 \%$ in Europe/North America (Swedish Cancer Foundation), and is also just a little higher than that of a screening of healthy individuals that ran in Hunan province, P.R. China, between 2005 and 2007 ( $n=11,278,0.5 \%$ STK1positive, unpublished data). The STK1-positive healthy individuals in this study did not suffer from any cancer diseases. However, two had hepatitis infection and one had liver disease (inflammation). These illnesses activate the immunological system, elevating a number of growth factors, such as EGF, TGFa, TGFb, PDGF, FGF, IGF-1 and THF (30). Hepatitis B and $\mathrm{C}$ as well as hepatic cirrhosis increase the risk of developing liver cell carcinoma (30). The lack of malignancies may be explained by the low mean age of 27 years in this group. It is known that the risk of developing carcinoma is elevated with increasing age (30), for example, in breast cancer the risk starts to be apparent after the age of 50 (29).

Based on our clinical experiences using STK1 to date, we suggest that individuals with a STK1 level just above the threshold value $(2 \mathrm{pM})$ should be retested within a year. It is also important that the evaluation of the STK1 level is interpreted together with a detailed history of the individual condition of the patient.

In conclusion, the STK1 marker shows high specificity and sensitivity, and is thus a reliable marker for the screening of healthy individuals. STK1 is also useful for prognosis and for monitoring the outcome of tumor therapy, in particular for the early prediction of progression and the recurrence of cancer diseases. It aids in making decisions, allowing for individualized therapy for different carcinomas and avoiding over-treatment and/or changes in treatment strategy. The STK1 assay may enable increased survival and, therefore, the quality of life of cancer patients. The STK1 marker is also a useful tool for the screening for and early prediction of the risk of cancer diseases, especially in areas with a higher frequency of tumor disease.

\section{Acknowledgements}

This investigation was supported by the Zhejiang Cancer Hospital, the Hangzhou First People's Hospital, the Zhejiang
Provincial People's Hospital, The Second Affiliated Hospital of Wenzhou Medical College and the Zhejiang Centre for Disease Control. We are deeply grateful to professor Zhang Ming at the Medical Institute of Zhejiang university, Mr. Zheng Mingchun at Zhejiang Deri Medical Tech. Ltd., Zheng Li, Zhang Ming, Jing Lin and Sino-Swed Tongkang Biotech Inc., Shenzhen, for their invaluable collaboration.

\section{References}

1. Tumor Markers. Eissa S (ed). Chapman \& Hall, New York, pp94-102, pp134-137, 1998.

2. Tocchi A, Costa G, Lepre L, Liotta G, Mazzoni G, Cianetti A and Vannini P: The role of serum and gastric juice levels of carcinoembryonic antigen, CA19.9 and CA72.4 in patients with gastric cancer. J Cancer Res Clin Oncol 124: 450-455, 1998.

3. Ychou M, Duffour J, Kramar A, Gourgou S and Grenier J: Clinical significance and prognostic value of CA72-4 compared with CEA and CA19-9 in patients with gastric cancer. Dis Markers 16: 105-110, 2000.

4. Dehaghani AS, Ghiam AF, Hosseini M, Mansouri S and Ghaderi A: Factors influencing serum concentration of CA125 and CA15-3 in Iranian healthy postmenopausal women. Pathol Oncol Res. 13: 360-364, 2007.

5. Pang RW, Joh JW, Johnson PJ, Monden M, Pawlik TM and Poon RT: Biology of hepatocellular carcinoma. Ann Surg Oncol 15: 962-971, 2008.

6. Ulmert D, Cronin AM, Bjork Th, O'Brien MF, Scardino PT, Eastam JA, Becker Ch, Berglund G, Vickers AJ and Lija H: Prostatic-specific antigen at or before age 50 as a predictor of advanced prostate cancer diagnosed up to 25 years later: a casecontrol study. BMC Med 6: 6, doi: 10.1186/1741-7015-6-6, 2008.

7. Cheung KL, Evans AJ and Robertson JF: The use of blood tumour markers in the monitoring of metastatic breast cancer unassessable for response to systemic therapy. Breast Cancer Res Treat 67: 273-278, 2001.

8. Hwa HL, Kuo WH, Chang LY, Wang MY, Tung TH, Chang KJ and Hsieh FJ: Prediction of breast cancer and lymph node metastatic status with tumour markers using logistic regression models. J Eval Clin Pract 14: 275-280, 2008.

9. Locker GY, Hamilton S, Harris J, Jessup JM, Kemeny N, Macdonald JS, Somerfield MR, Hayes DF and Bast RC Jr: ASCO 2006 update of recommendations for the use of tumor markers in gastrointestinal cancer. J Clin Oncol 24: 5313-5327, 2006.

10. He Q, Skog S and Tribukait B: Cell cycle related studies on thymidine kinase and its isoenzymes in Ehrlich ascites tumours. Cell Prolif 24: 3-14, 1991.

11. Kauffman MG and Kelly TJ: Cell cycle regulation of thymidine kinase: residues near the carboxyl terminus are essential for the specific degradation of the enzyme at mitosis. Mol Cell Biol 11: 2538-2546, 1991

12. Ke PY and Chang ZF: Mitotic degradation of human thymidine kinase 1 is dependent on the anaphase-promoting complex/ cyclosome-CDH1-mediated pathway. Mol Cell Biol 24: 514-526, 2004.

13. He Q, Wang N, Skog S, Ericsson S and Tribukait B: Characterization of a peptide antibody against a C-terminal part of human and mouse cytosolic thymidine kinase, which is a marker for cell proliferation. Eur J Cell Biol 70: 117-124, 1996.

14. Gronowitz JS, Hagberg H, Kallander CF and Simonsson B: The use of serum deoxythymidine kinase as a prognostic marker, and in the monitoring of patients with non-Hodgkin's lymphoma. Br J Cancer 47: 487-495, 1983.

15. O'Neill KL, Abram WP and McKenna PG: Serum thymidine kinase levels in cancer patients. Ir J Med Sci 155: 272-274, 1986.

16. Gronowitz JS, Bergström R, Nôu E, Påhlman S, Brodin O, Nilsson $S$ and Källander CF: Clinical and serologic markers of stage and prognosis in small cell lung cancer. A multivariate analysis. Cancer 66: 722-732, 1990.

17. Bresnick E, Mayfield ED Jr, Liebelt AG and Liebelt RA: Enzyme patterns in a group of transplantable mouse hepatomas of different growth rates. Cancer Res 31: 743-751, 1971.

18. He Q, Skog S, Fornander T, Johansson H, Johansson U, Hu G, Rutqvist LE and Skog S: Thymidine kinase 1 in serum predicts increased risk of distant or loco-regional recurrence following operation of early breast cancer patients. Anticancer Res 26: 4753-4760, 2006. 
19. Broët P, Romain S, Daver A, Ricolleau G, Quillien V, Rallet A, Asselain B, Martin PM and Spyratos F: Thymidine kinase as a proliferative marker: clinical relevance in 1,692 primary breast cancer patients. J Clin Oncol 19: 2778-2787, 2001.

20. Foekens JA, Romain S, Look MP, Martin PM and Klijn JG: Thymidine kinase and thymidylate synthase in advanced breast cancer: response to tamoxifen and chemotherapy. Cancer Res 61: 1421-1425, 2001

21. He Q, Zhang P, Zou L, Li HX, Wang XQ, Zou S, Fornander T and Skog S: Concentration of thymidine kinase 1 in serum (S-TK1) is a more sensitive proliferation marker in human solid tumors than its activity. Oncol Rep 14: 1013-1019, 2005.

22. Greene FL, Page DL, Fleming ID, Fritz AG, Balch CM, Haller DG and Morrow M. AJCC Cancer Staging Manual. Springer-Verlag, New York, 2002.

23. Wu CJ, Yang RJ, Zhou J, Bao S, Zou L, Mao YR and He Q: Production and characterisation of a novel chicken IgY antibody raised against $\mathrm{C}$-terminal peptide from human thymidine kinase 1 . J Immunol Methods 277: 157-169, 2003.

24. Sauer $\mathrm{H}$ and Wilmanns W: Thymidine kinase. In: Method of Enzymatic Analysis. Vol 3. Bergmeyer HU, Bergmeyer J and Grassl M (eds). Verlag Chemie, Weinheim, Deerfield Beach, Florida, pp 468-473, 1983.

25. He Q, Zou L, Zhang PA, Lui JX, Skog S and Fornander T: The clinical significance of thymidine kinase 1 measurement in serum of breast cancer patients using anti-TK1 antibody. Int J Biol Markers 15: 139-146, 2000.

26. Zou L, Zhang PG, Zou S, Li Y and He Q: The half-life of cytosolic thymidine kinase in serum by ECL dot blot: a potential marker for monitoring the response to surgery of patients with gastric cancer. Int J Biol Markers 17: 135-140, 2002.
27. Li HX, Zhang S, Lei DS, Wang XQ, Skog S and He Q: Serum thymidine kinase 1 (STK1) is a prognostic and monitoring factor in patients with non-small-cell lung cancer. Oncol Rep 13 145-149, 2005

28. Zhang J, Jia Q, Zou S, Zhang P, Zhang X, Skog S, Luo P, Zhang W and $\mathrm{He}$ Q: Thymidine kinase 1: a proliferation marker for determining prognosis and monitoring the surgical outcome of primary bladder carcinoma patients. Oncol Rep 15: 455-461, 2006.

29. Pathology and Genetics of Tumours of the Breast and Female Genital Organs. In: WHO Classification of Tumours. Tavassoli FA and Devilee P (eds). IARC Press, Lyon, pp63-73, 2003.

30. General and Systematic Pathology. 4th edition. Underwood JCE (ed). Churchill Livingstone, Edinburgh, 2004

31. Gronowitz JS, Källander FR, Diderholm H, Hagberg H and Pettersson U: Application of an in vitro assay for serum thymidine kinase: results on viral disease and malignancies in humans. Int $\mathbf{J}$ Cancer 33: 5-12, 1984.

32. Tanaka K, Sishido T, Morimoto M, Inoue S, Takamura Y and Masumura M: Elevated serum thymidine kinase activity in patients with acute viral hepatitis. Gastroenterol Jpn 28: 51-55, 1993.

33. Turriziani O, Butera O, Gianotti N, Parisi SG, Mazzi R, Girardi E, Iaiani G, Antonelli L, Lazzarin A and Antonelli G: Thymidine kinase and deoxycytidine kinase activity in mononuclear cells from antiretroviral-naive HIV-infected patients. AIDS 19: 473-479, 2005.

34. Sandström H, Wahlin A, Eriksson M and Bergström I: Serum thymidine kinase in congenital dyserythropoietic anaemia type III. Br J Haematol 87: 653-654, 1994.

35. Yang L, Parkin DM, Ferlay J, Li L and Chen Y: Chines cancer incident rate: estimates of cancer incidence in China for 2000 and projections for 2005. Cancer Epidemiol Biomarkers Prev 14: 243-250, 2005. 